\title{
A plant-based multicomponent vaccine protects mice from enteric diseases
}

\author{
Jie Yu and William H.R. Langridge*
}

\begin{abstract}
Cholera toxin (CT) B and A2 subunit complementary DNAs (cDNAs) were fused to a rotavirus enterotoxin and enterotoxigenic Escherichia coli fimbrial antigen genes and transferred into potato. Immunoblot and enzymelinked immunosorbent assay (ELISA) results indicated that the fusion antigens were synthesized in transformed tuber tissues and assembled into cholera holotoxin-like structures that retained enterocyte-binding affinity. Orally immunized mice generated detectable levels of serum and intestinal antibodies against the pathogen antigens. Elevated levels of interleukin 2 (IL2) and interferon $\gamma$ (INF $\gamma$ ) detected in immunogenchallenged spleen cells from the immunized mice indicated the presence of a strong Th1 immune response to the three plant-synthesized antigens. This result was supported by flow cytometry analysis of immunized mouse spleen cells that showed a significant increase in $\mathrm{CD}^{+}$lymphocyte numbers. Diarrhea symptoms were reduced in severity and duration in passively immunized mouse neonates following rotavirus challenge. The results suggest that food plants can function as vaccines for simultaneous protection against infectious virus and bacterial diseases.
\end{abstract}

Acute gastroenteritis is second only to acute respiratory disease as a cause of death worldwide ${ }^{1}$. Cholera, rotavirus, and enterotoxigenic E. coli (ETEC) are the three major causative agents of acute infectious enteric diseases. Recently, edible plants have been used successfully as production and delivery systems for pathogen antigens $^{2-5}$. The plant-expressed antigens generated protective antibodies in orally immunized animals ${ }^{6-8}$. However, the relatively low expression levels of foreign antigens synthesized in transgenic plants, coupled with the modest immune response following oral immunization, remain limiting factors for development of an effective plant-based vaccine. The recent expression and successful assembly of cholera toxin B subunits into biologically active oligomeric structures in transgenic potato plants ${ }^{4}$ provides a valuable approach for targeting plant-synthesized antigens to the gut-associated lymphoid tissues. Cholera toxin possesses distinct carrier and adjuvant properties. Antigens genetically fused to CTA or CTB subunit were found to stimulate strong immune response in orally immunized animals ${ }^{9,10}$. In this report, we have expressed a cholera toxin fusion with two different disease antigens in transformed potatoes. In a C-terminal fusion, we linked the 22-amino acid immunodominant epitope of the murine rotavirus enterotoxin NSP4 to the CTB subunit, and in a N-terminal fusion, we fused the ETEC fimbrial colonization factor CFA/I to the CTA2 subunit. Following Agrobacterium tumefaciens transformation of potato leaf tissue with a plant expression vector carrying the two cholera toxin fusion genes, we regenerated transformed plants. Female CD-1 mice were immunized by feeding transformed potato tuber tissues containing the cholera toxin fusion proteins. The titers of serum IgG and intestinal IgG and IgA antibodies against NSP4, CTB, and CFA/I were measured in the immunized mice. Spleen cells from immunized mice were analyzed for IL2, IL4, and INF $\gamma$ cytokine secretion, and for T-lymphocyte subpopulations. Passively immunized mouse pups from orally immunized dams were challenged with SA-11 rotavirus to determine the level of protection against rotavirus infection delivered by the plantbased vaccine.

\section{Results and discussion}

Expression of CFA/I-CTA2 and CTB-NSP4 fusion genes. The CTB-NSP4 fusion gene was cloned into plant expression vector pPCV701 under control of the mas $\mathrm{P} 2$ promoter. The CFA/I-CTA2 fusion gene was cloned downstream of mas P1 promoter. Each cholera toxin fusion gene contains its own leader sequence and an endoplasmic reticulum (ER) retention signal. To increase the flexibility of the fusion protein, a four-amino acid GPGP hinge region was inserted between the CTB and NSP4 peptides (Fig. 1A). Transformed potato plants were generated from leaf explants following an $A$. tumefaciens-meditated stable transformation method ${ }^{4}$.

Bacterial CTB (Fig. 1B) assembled into an oligomeric structure with a molecular weight of $45 \mathrm{kDa}$, characteristic of the CTB pentamer. Plant-produced CTB-NSP4 fusion peptide (Fig. 1B) formed a $50 \mathrm{kDa}$ oligomeric structure. The $5 \mathrm{kDa}$ increase in molecular weight is consistent with the presence of the additional NSP4 peptide and the six-amino acid SEKDEL signal. The plant sample containing both CFA/I-CTA2 and CTB-NSP4 fusion proteins (Fig. 1B) showed the presence of a $70 \mathrm{kDa}$ protein band, indicative of the insertion of CFA/I-CTA2 peptide into the CTB-NSP4 oligomer. The untransformed plant (Fig. 1B) showed no cross reaction with the cholera toxin antibody. Immersion of the samples in boiling water for $5 \mathrm{~min}$ resulted in dissociation of the multimeric structures into monomeric peptides (Fig. 1C). The bacterial CTB monomer was found to have a molecular weight of $11 \mathrm{kDa}$. The plant-derived CTB-NSP4 multimer dissociated into an $18 \mathrm{kDa}$ monomer consistent with the molecular weight of CTB plus NSP4. The apparent molecular weight of the pentamer is smaller than expected, because it is not significantly denatured before protein gel separation. Therefore, due to its globular shape, the mobility of the oligomeric structure is faster than expected for the sum of its parts, hence the lower molecular weight.

Induction of serum and intestinal antibodies in orally immunized mice. Following five oral inoculations with transgenic potato tuber tissues, blood samples were collected and the serum anti-CTB,

Department of Biochemistry and Center for Molecular Biology and Gene Therapy, Loma Linda University, Loma Linda, CA 92350. ${ }^{*}$ Corresponding author (blangridge@som.llu.edu). 
A

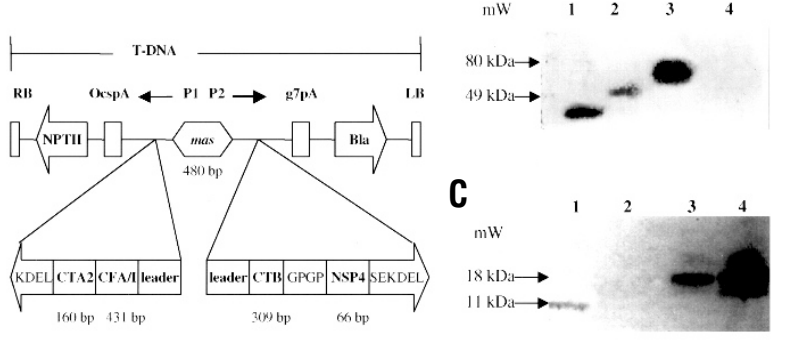

Figure 1. Expression of cholera toxin fusion proteins in transgenic potato. (A) Plant expression vector pPCV701 CFA/I-CTB-NSP4. The vector contains all four genes located within the transferred DNA (T-DNA) sequence, which is flanked by the right and left border ( $R B$ and LB). The CTBH:NSP4 (114-135):SEKDEL coding sequence is under control of the mas $\mathrm{P} 2$ promoter. The CFA/l:CTA2 coding sequence is under control of the mas $\mathrm{P} 1$ promoter. The neomycin phosphotransferase II gene (NPT II) provides resistance to kanamycin for selection of transformed plants. The $\beta$-lactamase gene (Bla) provides resistance to ampicillin for selection in E. coli and carbenicillin for selection in A. tumefaciens. The g7pA polyadenylation signal is from the $A$. tumefaciens $\mathrm{T}_{\mathrm{L}}$-DNA gene 7 , and the OcspA polyadenylation signal is from the octopine synthase gene. (B) Immunoblot detection of the oligomeric CTB-NSP4 and CTA2-CFA/ fusion proteins. The oligomeric CTB-NSP4 fusion protein was detected with anti-CTB antibody as the primary antibody and anti-mouse IgG conjugated to alkaline phosphatase as the secondary antibody. Lane 1, $100 \mathrm{ng}$ commercial bacterial CTB pentamer; lane 2, $100 \mu \mathrm{g}$ total soluble protein (TSP) from potato plants transformed with the CTB-NSP4 fusion gene; lane 3, $100 \mu \mathrm{g}$ TSP from potato plants transformed with both CTB-NSP4 and CFA/l-CTA2 fusion genes; lane 4, $100 \mu \mathrm{g}$ TSP from an untransformed potato plant. (C) Immunoblot detection of monomeric CTB-NSP4 fusion protein with anti-CT antiserum after boiling the plant homogenates for $5 \mathrm{~min}$ in sodium dodecyl sulfate (SDS) containing buffer Lane 1, $100 \mathrm{ng}$ boiled commercial bacterial CTB monomer; lane 2, $100 \mu \mathrm{g}$ boiled TSP from an untransformed potato plant; lane 3,100 $\mu \mathrm{g}$ of boiled TSP from potato plants transformed with the CTB-NSP4 gene; lane 4 $100 \mu \mathrm{g}$ boiled TSP from potato plants transformed with both CTB-NSP4 and CFA/I-CTA2 genes.

-NSP4, and -CFA/I IgG titers were measured by ELISA. Out of 10 mice, 8 generated serum IgG against CTB with a mean titer of 312.5 \pm 81.3 . Of the 10 immunized mice, 8 developed serum IgG against NSP4 with a mean titer of $125 \pm 61.23$, and 10 developed serum IgG against CFA/I with a mean titer of $84 \pm 44.2$ (Fig. 2A).

Intestinal IgG and IgA antibody titers against the three antigens were analyzed by chemiluminescent ELISA method. Out of 10 immunized mice, 5 generated measurable intestinal anti-CTB antibody titers; out of 10 immunized mice, 5 were found to have measurable intestinal anti-SP4 antibody titers, and 6 out of 10 mice were found to have significant intestinal anti-FA/I antibody titers (Fig. 2B). Of 10 immunized mice, 4 developed mucosal IgG and IgA antibodies against all three antigens. The intestinal antibody titers were relatively low in comparison with serum IgG levels. Mice fed untransformed potato tuber tissues did not develop detectable specific serum or mucosal antibodies (Fig. 2). Since CTB pentamers can bind to $\mathrm{G}_{\mathrm{M} 1}$ ganglioside located on the mucosal epithelial cell surface, induction of both systemic and mucosal antibodies in the immunized mice indicated successful delivery of the cholera toxin fusion proteins to the gut-associated lymphoid tissues.

Adjuvant and carrier functions of CTB in the CTB-NSP4 fusion protein were determined by measuring serum anti-NSP4 antibody titers in mice from different vaccination groups. Mice fed the NSP4 peptide alone generated the lowest anti-NSP4 titer. Immunization with $10 \mu \mathrm{g}$ of bacterial CTB (the same amount detected in the plantderived CTB-NSP4 fusion protein) increased the serum anti-NSP4 IgG titer approximately twofold. Mice fed $3 \mathrm{~g}$ transformed potato tuber tissues containing the CTB-NSP4 fusion protein developed the
A

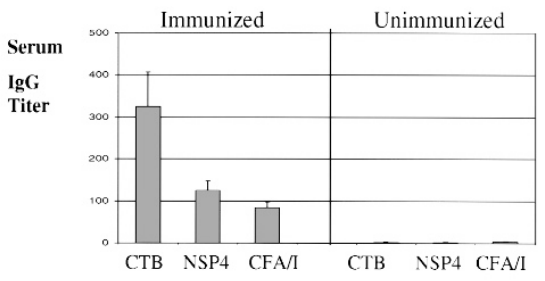

B

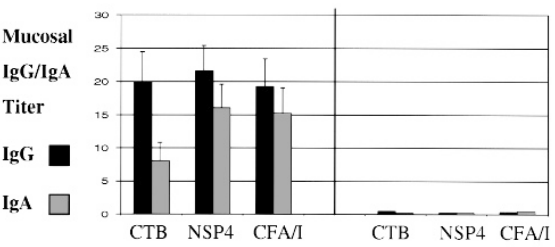

Figure 2. Measurement of anti-CTB, NSP4, and CFA/l antibody titers in mice after oral immunization with transgenic potato tuber tissues. CD-1 mice were fed transgenic potato tissues containing $10 \mu \mathrm{g}$ of CTB-NSP4 and CFA/I-CTA2 fusion proteins five times at one-week intervals. Mice fed equal amounts of untransformed potato tissues served as a negative control. (A) Serum IgG antibody titers against CTB, NSP4, and CFA/l proteins. (B) Intestinal IgG and IgA antibody titers against CTB, NSP4, and CFA/l proteins. Antibody titers were determined by chemiluminescent ELISA method. The antibody titer was defined as the reciprocal of the highest dilution of the serum or intestinal contents sample that generated a luminescence signal above the background levels.

highest anti-NSP4 titer (Fig. 3). Small soluble proteins like the NSP4 22-amino acid epitope that are highly immunogenic by parenteral routes are frequently ineffective when administered orally unless a large dose of the protein is used ${ }^{11,12}$. This result can be attributed to intestinal digestion and lack of tropism of the peptide for the gutassociated lymphoid tissues. Either cholera holotoxin or the CTB subunit, which function as mucosal adjuvants, can stimulate an immune response against co-administered protein antigens ${ }^{13-16}$. Directly linking small antigens with CTB subunit not only results in specific targeting of the antigens to the mucosal immune system through specific enterocyte attachment but also increases the local antigen concentration at the mucosal surface, which may explain the strength of the immune response directed against the CTB-NSP4 fusion protein.

Cytokine assays and detection of CD4 memory cells. Following multiple oral immunizations, the IL2 and the INFy expression levels in spleen cells dramatically increased, reaching the highest level 34 days after the fifth immunization and decreasing to basal levels by 68 days after vaccination. Throughout this time period IL4 levels remained low, equivalent to those found in unimmunized mice (Fig. 4). Thus, cytokine expression pattern clearly indicated a Th1 lymphocyte-mediated immune response generated by feeding mice the plant-derived cholera toxin fusion antigens. Cholera toxin and the CTB subunit function as potent adjuvants and can promote antigen priming of both Th1 and Th2 CD4 ${ }^{+} \mathrm{T}$-precursor cells ${ }^{17,18}$. The pattern of the T-helper lymphocyte response may depend on the nature of the co-administered antigens ${ }^{19}$. Oral immunization with CTB-insulin or CTB-glutamate decarboxylase fusion proteins generate a Th2 response, whereas oral immunization with recombinant E. coli CFA/I stimulated a biphasic Th-lymphocyte response: a predominant Th2 response at the early stage, and then a Th1 celldominant response four weeks after immunization ${ }^{20,21}$. The rotavirus nonstructural protein NSP4, which acts as a viral enterotoxin, stimulated increased production of IL2 and INF $\gamma$ in humans ${ }^{22}$. Therefore, we were not surprised to see the overall cytokine secretion pattern of this multicomponent plant vaccine in the mouse model to be indicative of a strong Th1 response. 


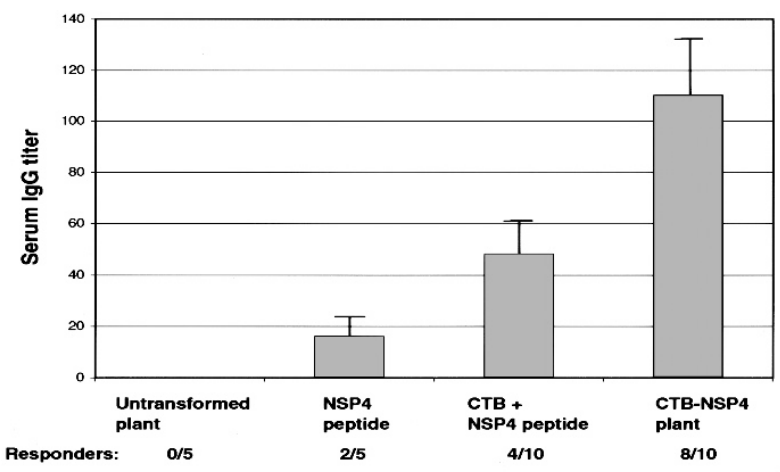

Figure 3. The adjuvant and carrier functions of CTB in CTB-NSP4 fusion protein. Serum IgG antibody titers against the NSP4 peptide were measured following mucosal immunization of mice from selected vaccination groups. From left to right, group 1 was composed of mice fed $3 \mathrm{~g}$ of untransformed potato tuber tissues, group 2 of mice fed $10 \mu \mathrm{g}$ of synthetic 22-amino acid NSP4 peptide, group 3 of mice fed $10 \mu \mathrm{g}$ NSP4 synthetic peptide mixed with $10 \mu \mathrm{g}$ of commercial bacterial CTB, and group 4 mice fed $3 \mathrm{~g}$ of transgenic potato tuber tissues containing $\sim 10 \mu \mathrm{g}$ CTB-NSP4 fusion protein. Responders: The number of mice that developed a detectable serum IgG titer against NSP4 peptide in each group divided by the total number of mice immunized.

Flow cytometry analysis of spleen cells collected on days 13, 34, and 68 after the last vaccination showed an elevated population of $\mathrm{CD}^{+}$memory cells in comparison with the unimmunized mice for two months after immunization (Fig. 5C). The subpopulation of $\mathrm{CD}^{+}$memory cells (CD62- ${ }^{-} \mathrm{CD} 44^{+}$, gate $\left.\mathrm{R} 4\right)$ detected in the immunized mice (Fig. 5B) was significantly higher than the $\mathrm{CD} 4^{+}$memory cell subset in unimmunized mice (Fig. 5A). The significantly increased T-helper memory cell population in immunized mice indicated successful protective immunization mediated by the plantdelivered virus and bacterial antigens. The presence of increased numbers of memory lymphocytes provided the ability to mount a strong immune response following a second encounter with the same pathogen. Splenocyte flow cytometry experiments did not show a significant increase in $\mathrm{CD} 8^{+}$memory cells in comparison with unimmunized mice (data not shown). This result may be explained by the substantial cytotoxic T-lymphocyte (CTL) population present in the spleen of immunized mice, of which the subpopulation of antigen-specific CD8 ${ }^{+}$memory cells would have been only a small component. Alternatively, a temporary weak cytotoxic immune response is often characteristic of oral immunization with individual recombinant antigens 9,23 . In future experiments, the antigen-activated spleen cells will be incubated with effector cells containing ${ }^{51} \mathrm{Cr}$ to determine the extent of CTL activity.

Rotavirus challenge and diarrhea reduction. Anti-NSP4 antibody protection against rotavirus-induced diarrhea was evaluated by virus challenge of pups born to dams immunized with the plant-derived CTB-NSP4 fusion protein ${ }^{24}$. The number of pups that developed diarrhea symptoms and the duration of the diarrhea was significantly reduced in neonates passively immunized with CTB-NSP4 fusion protein in comparison with pups born to unimmunized dams. On day 3 after rotavirus challenge, a 50\% reduction of diarrhea symptoms was detected in the immunized pups. Complete resolution of diarrhea symptoms occurred four days after virus challenge in pups from immunized dams (Fig. 6, the third column in each group). To exclude the possibility of diarrhea reduction due to the presence of anti-CTB antibodies, pups born to dams immunized with plantderived CTB only were also challenged with an identical dose of rotavirus SA11 (Fig. 6, second column in each group). No reduction

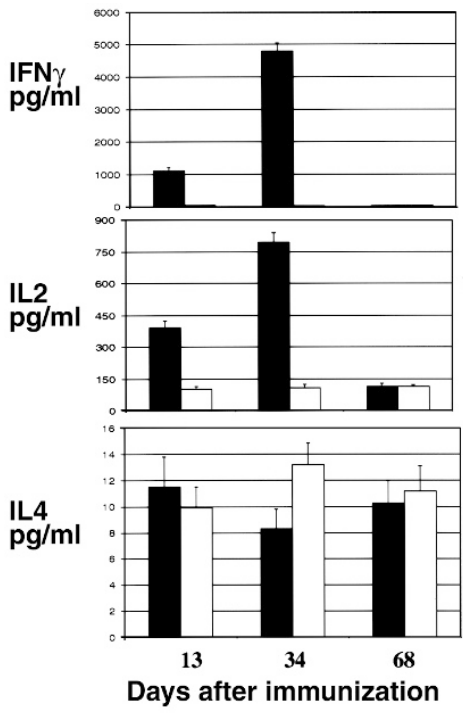

Figure 4. Measurement of cytokine levels in spleen cells from mice immunized with potato tissues containing CTB, NSP4, and CFA/I proteins. Spleen cells were isolated from unimmunized CD-1 mice and mice immunized with potato tuber tissues containing CTB-NSP4 and CFA/I-CTA2 antigens. Supernatants from the spleen cell culture were assayed for the presence of IL2, IL4, and INF $\gamma$ levels on days 13, 34, and 68 after the final oral immunization. The levels of IL2, INF $\gamma$, and IL4 were defined as the amount of the cytokines produced in $1 \mathrm{ml}$ spleen cell culture $\left(3 \times 10^{6}\right.$ cells). White bars represent the cytokine levels of unimmunized mice. Black bars represent the cytokine levels of mice orally immunized with tuber tissues containing CTB-NSP4 and CFA/I-CTA2 antigens.

of diarrhea symptoms was detected in mice immunized with plantderived CTB alone. This experiment demonstrated that anti-NSP4 antibodies generated in orally immunized mice were passed on to the pups and provided protection from the onset, as well as significantly reducing the duration of rotavirus infection.

Development of plant-based multicomponent oral vaccines. Mucosal vaccines capable of inducing both humoral and mucosal immunity would provide the most ideal defense against infectious enteric diseases. Most soluble protein antigens are sensitive to $\mathrm{pH}$ and enzymatic digestion in the stomach and intestinal tract, howev$\mathrm{er}^{1}$. Yet cholera toxin is a remarkably stable protein and a potent immunogen that has been successfully synthesized in bacterial or viral systems as a carrier molecule for the transfer of chemically or genetically fused antigens ${ }^{25,26}$. Initial mucosal immunization experiments, in which synthesis of the CTB subunit in transgenic potato was followed by oral immunization of mice with transformed plant tissues, demonstrated a significant protective effect of the plantbased vaccine and has opened up development of effective mucosal vaccines in stably transformed plants ${ }^{8}$. Based on this result, cholera toxin fusion proteins with other antigens expressed in plants became the next logical focus for the development of food plant-based oral vaccines. Thus the additional finding that the cholera toxin A2 subunit-CFA/I fusion protein associates with the cholera toxin B subunits to form a holotoxin-like oligomer capable of binding to gangliosides present in the enterocyte membrane now provides us with the opportunity to generate multicomponent vaccines in plants in which several antigens can be targeted to the mucosal immune system for greater protection efficacy. Oral inoculation of CD1 mice with the multicomponent plant vaccine induced significant levels of humoral and mucosal antibodies against CTB, NSP4, and CFA/I antigens. Furthermore, the anti-NSP4 antibodies 
A
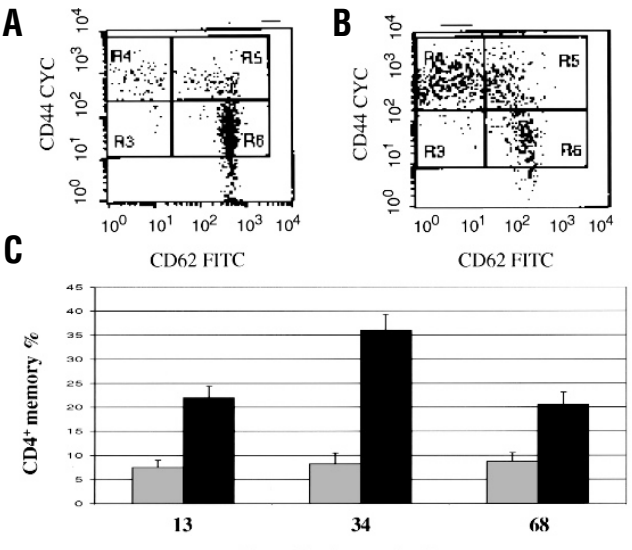

Days after immunization

Figure 5. Detection of $\mathrm{CD} 4^{+}$memory cells in spleen cells from mice immunized with transgenic potato tissues. Flow cytometry analysis of $\mathrm{CD}^{+}{ }^{+}$-cell subpopulations isolated from the spleen of $(\mathrm{A})$ unimmunized CD-1 mice and (B) mice immunized with potato tissues containing CTB-NSP4 and CFA/I-CTA2 fusion proteins. Cells were stained with FITC-conjugated anti-CD62 and CYC-conjugated anti-CD44 mAbs as described in the Experimental Protocol. The chart scale is $\log _{10}$ of the fluorescence intensity. (C) Analysis of $\mathrm{CD} 4^{+}$memory cells in the spleen of immunized mice. Spleen cells were collected at 13,34 , and 68 days following the last immunization. Gray column represents the percentage of memory cells in mice fed untransformed potato tissues, and the black column indicates the percentage of memory cells in mice fed transgenic potato tissues.

in passively immunized mouse pups offered protection against rotavirus challenge.

Cholera toxin neutralization assays and mouse ileal loop ligation assays previously performed in our laboratory on mice immunized with potato-synthesized CTB provided a significant protective effect against diarrhea generated by cholera toxin ${ }^{8}$. The protective efficacy studies with cholera toxin were not repeated because, in our present animal immunization study, the anti-CTB antibody titer detected was fivefold higher than obtained in our previous experiments.

T-helper lymphocytes play important roles in generating antigenspecific humoral and cell-mediated immune responses. Serum and mucosal antibodies can be induced either through Th1- or Th2-cell responses, depending on the nature of the antigen peptide presented to lymphocyte in the lamina propria of the intestinal tract ${ }^{19}$. Cytokine assays performed on spleen cells isolated from immunized mice indicated the presence of a strong Th1-lymphocyte response, possibly including cytolytic T cells. Detection of a Th1 response may be due to the presence of a viral antigen NSP4 in the CTB fusion protein ${ }^{27}$. The observed Th1 response correlated with the appearance of modest titers of mucosal antibodies. Detection of lymphocyte populations from immunized mice by flow cytometry showed a stable T-helper memory cell population two months after vaccination. Following immunization, the $\mathrm{CD}^{+}$cell population remained unchanged in the spleen cell flow cytometry analysis. Previous cellsorting experiments indicate that the cytotoxic $\mathrm{T}$-cell response in the spleen may not be a good indicator of the localized cytotoxic T-cell response at the mucosal surface ${ }^{28}$. The expression of multiple antigens in individual transgenic plants and the significant antibody titer and protective immune response generated against all of the antigens by the mammalian mucosal immune system in response to mucosal immunization with transformed plant tissues creates opportunities for production of food plant-based vaccines with increased protective efficacy against individual virus or bacterial pathogens (multivalent vaccine) or multiple pathogens (multicomponent vaccines).

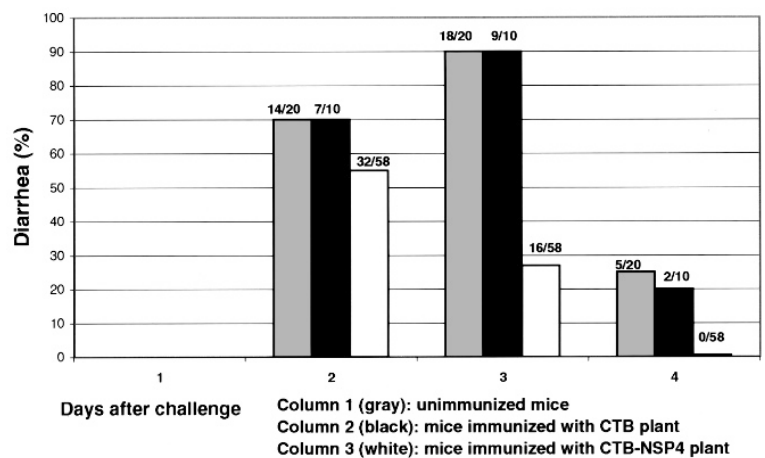

Figure 6. Protection against rotavirus infection in passively immunized mouse pups. Mouse pups born to CD-1 mouse dams orally immunized with transformed potato tubers containing the CTB-NSP4 fusion protein were challenged with $15 \times \mathrm{DD}_{50}$ dose of rotavirus strain SA11. The vertical axis represents the percentage of mouse pups that developed diarrhea from day 1 to day 4 following virus challenge. The number of pups that developed diarrhea in comparison to the total number of pups in each group is indicated on top of each column. The first column in each group (gray) indicates the pups born to dams fed untransformed potato tissues. The second column (black) represents the pups born to dams fed transgenic potato tissues containing CTB protein only. The third column (white) represents the pups born to dams fed transgenic potato tissues containing the CTB-NSP4 fusion protein.

\section{Experimental protocol}

Plant expression vector construction and plant transformation. The plant expression vector pPCV701CFA/I-CTB-NSP4 was assembled from the parental plasmid pPCV701 in the following manner. A nucleotide sequence encoding the ER retention signal SEKDEL was first cloned into the plant expression vector pPCV701 3' to the P2 site of the mannopine synthase (mas) dual P1, P2 promoter. The CTB gene and its leader sequence were amplified by PCR from the cholera toxin (ctxAB) operon in plasmid pPT42 (provided by J. Mekalanos, Harvard University School of Medicine). The CTB 3'-primer was designed to contain an oligonucleotide encoding the tetrapeptide hinge (GPGP) to incorporate a degree of flexibility between the CTB and NSP4 peptides. A synthesized DNA fragment encoding the rotavirus enterotoxin NSP4 (114-135) epitope was inserted in frame between the CTB hinge and the SEKDEL sequences. The CTA leader sequence and the CTA2 gene were amplified by PCR from the ctxAB operon and cloned into PPCV701 3' immediately downstream of the mas $\mathrm{P} 1$ promoter region. A DNA fragment encoding the ETEC colonization factor CFA/I (431 bp) was amplified from plasmid pIGx15A (provided by Dr. M. Levine, University of Maryland), and was inserted in frame between the CTA leader sequence and the CTA2 gene. Detailed cloning descriptions and primer sequences are available upon request. The resultant plant expression vector pPCV701CFA/I-CTB-NSP4 was introduced into A. tumefaciens strain GV3101 pMP90RK. Potato (Solanum tuberosum cv. Bintje) leaf tissue explants were transformed with A. tumefaciens harboring the plant expression vector pPCV701 CFA/I-CTBNSP4. Transformed plants were regenerated from the explants on selection medium containing kanamycin. Before analysis of antigen gene expression, transgenic tubers were stimulated to produce high levels of the antigen proteins by incubation of tuber slices on growth medium containing auxin 2,4-D for four days at room temperature.

Immunoblot detection of the CFA/I and CTB-NSP4 fusion proteins. Protein extracts from auxin stimulated transformed potato tubers containing $100 \mu \mathrm{g}$ of total soluble protein (TSP) were loaded on a $10-15 \%$ sodium dodecyl sulfate-polyacrylamide gel electrophoresis (SDS-PAGE) gel with or without $5 \mathrm{~min}$ boiling before electrophoresis. The separated protein bands were transferred to nitrocellulose membrane by electroblotting on a semi-dry blotter (Sigma Chemical Co., St. Louis, MO) at $30 \mathrm{~V}, 60 \mathrm{~mA}$ for $90 \mathrm{~min}$. The locations of CTB, NSP4, and CFA/I proteins were identified by incubation of the blot in rabbit anti-CTB antiserum (Sigma; 1:5,000 dilution) overnight at room temperature followed by incubation in alkaline phosphatase-conjugated mouse 
anti-rabbit IgG (Sigma; 1:10,000 dilution) for $2 \mathrm{~h}$ at room temperature. Finally, the membrane was incubated in the substrate BCIP/NPT (Sigma) for $10 \mathrm{~min}$.

Mouse immunization and cell cultures. A group of $10 \mathrm{CD}-1$ female mice were each fed, on days $0,5,15,23$, and 56 of the experiment, $3 \mathrm{~g}$ trangenic potato tuber tissues containing a total of $10 \mu \mathrm{g}$ of the recombinant fusion proteins previously determined by chemiluminescent ELISA. Using the same feeding schedule, a group of $5 \mathrm{CD}-1$ mice were each fed $3 \mathrm{~g}$ of untransformed potato tuber tissues as a negative control. To evaluate the adjuvant effect of the CTB protein in the CTB-NSP4 fusion, CD1 mice ( 5 per group) were gavaged with pure NSP4 peptide with or without pure bacterial CTB (adjuvant) according to the same oral inoculation schedule. On day 13 after the final immunization, blood was taken from each mouse for serum antibody titer determination. Three mice per group were euthanized at three different time points: 13, 34, and 68 days after the fifth immunization. Intestinal washings were collected for mucosal IgA antibody detection. Spleen cells from both immunized and negative control mice $\left(3 \times 10^{6}\right.$ cells/well $)$ were suspended in RPMI 1640 medium containing 10\% FCS in duplicate samples in 24-well tissue culture plates. After incubation for $72 \mathrm{~h}$ at $37^{\circ} \mathrm{C}$ in a humidified, $5 \% \mathrm{CO}_{2}$ incubator, supernatants from the spleen cell cultures were collected for quantification of IL2, INF $\gamma$, and IL4 secretion.

Cytokine and flow-cytometric analysis of T-lymphocyte populations in immunized mice. Spleen cell culture supernatants were assayed by ELISA for IL2, IL4, and INF $\gamma$ production (kits supplied by Endogen, Inc., Woburn, MA). Spleen lymphocytes were stained with fluorochrome-labeled monoclonal antibodies (mAbs) for immunophenotyping. Two $\mathrm{mAb}$ panels were constructed for three-color analysis: fluorescein isothiocyanate (FITC), phycoerythrin (PE), and Cy-Chrome. The first combination used, $\mathrm{CD} 62 \mathrm{~L}^{\star} \mathrm{FITC} / \mathrm{CD} 4{ }^{\star} \mathrm{PE} / \mathrm{CD} 44^{\star} \mathrm{Cy}$-Chrome, designates naïve and memory T-helper cells. The second combination, CD62 ${ }^{\star} \mathrm{FITC} / \mathrm{CD} 8 \mathrm{~b} .2^{\star} \mathrm{PE} / \mathrm{CD} 44^{*} \mathrm{Cy}-$ Chrome, designates naïve and memory cytotoxic $\mathrm{T}$ cells. The spleen cells were resuspended at $10^{6}$ cells $/ \mathrm{ml}$ in PBS and stained with fluorochrome-labeled
mAbs. The labeled cells were analyzed by flow cytometry to determine the T-lymphocyte memory cell subpopulations.

Evaluation of protection against rotavirus challenge. Adult female CD-1 mice (five per group) were fed $3 \mathrm{~g}$ of untransformed or trangenic potato tuber slices once a week for four weeks. Immediately following the fourth immunization at maximum anti-NSP4 antibody titer, the mice were mated with uninfected males. After a 19- to 20-day gestation period, mouse pups were born to the immunized dams. On day 6 post parturition, each pup received one oral dose of simian rotavirus SA-11 in $50 \mu \mathrm{PBS}$ that contained $15 \mathrm{DD}_{50}$ (the virus dose determined empirically to cause diarrhea in $50 \%$ of the mouse pups). The mice were examined for the presence of diarrhea daily for five days following inoculation by gentle palpation of their abdomen to produce fecal pellets. The diarrhea score and the proportion of mice showing diarrhea symptoms in each study group were recorded.

Acknowledgments

We would like to thank Dr. John Mekalanos (Harvard University School of Medicine) for the gift of the CTX gene, Dr. Mary Estes and Dr. Carl Zeng (Baylor School of Medicine) for providing rotavirus antiserum specific for the NSP4 22-amino acid epitope and rotavirus SA-11 for rotavirus challenge experiments, and for many helpful discussions. Further, we would like to thank Dr. Myron Levine (Center for Vaccine Development, University of Maryland School of Medicine) for providing us with the E. coli CFA/I gene and the CFA/I fimbrial protein. This work was supported in part by a subcontract to W.H.R.L. by the US Army and the National Medical Technology Testbed Inc. and by an intramural grant awarded to W.H.R.L. by Loma Linda University. The views, opinions, and/or findings contained in this report are strictly those of the authors and should not be construed as a position policy, decision, or endorsement of the federal government or of the National Medical Technology Testbed, Inc.

Received 7 December 2000; accepted 30 March 2001
1. Conner M.E. et al. Development of a mucosal rotaviurs vaccine. In Mucosal vaccines. (eds Kiyono, H., Ogra, P.L. \& McGhee, J.R.) 325-326 (Academic Press, San Diego, CA; 1996)

2. Mason, H.S., Lam, D.M. \& Arntzen, C.J. Expression of hepatitis B surface antigen in transgenic plants. Proc. Natl. Acad. Sci. USA 89, 11745-11749 (1992).

3. Mason, H.S. et al. Expression of Norwalk virus capsid protein in transgenic tobacco and potato and its immunogenicity in mice. Proc. Natl. Acad. Sci. USA 93 5335-5340 (1996).

4. Arakawa, T., Chong, D.K., Merritt, J.L. \& Langridge, W.H. Expression of cholera toxin B subunit ologomers in transgenic potato plants. Transgenic Res. $\mathbf{6}$, 403-413 (1997).

5. Modelska, A. et al. Immunization against rabies with plant-derived antigen. Proc. Natl. Acad. Sci.USA 95, 2481-2485 (1998).

6. Thanavala, Y., Yang, Y.F., Lyons, P., Mason, H.S. \& Arntzen, C.J. Immunogenicity of transgenic plant-derived hepatitis B surface antigen. Proc. Natl. Acad. Sci. USA 92, 3358-3361 (1995)

7. Haq, T.A., Mason, H.S., Clements, J.D. \& Arntzen, C.J. Oral immunization with a recombinant bacterial antigen produced in transgenic plants. Science 268, 714-716 (1995).

8. Arakawa, T., Chong, D.K. \& Langridge, W.H. Efficacy of a food plant-based oral cholera toxin B subunit vaccine. Nat. Biotechnol. 16, 292-297 (1998).

9. Czerkinsky, C., Russell, M.W., Lycke, N., Lindblad, M. \& Holmgren, J. Oral administration of a streptococcal antigen coupled to cholera toxin B subunit evokes strong antibody responses in salivary glands and extramucosal tissues. Infect. Immun. 57, 1072-1077 (1989).

10. Holmgren, J., Lycke, N. \& Czerkinsky, C. Cholera toxin and cholera B subunit as oral-mucosal adjuvant and antigen vector systems. Vaccine 11, 1179-1184 (1993).

11. Bloom, P.D. \& Boedeker, E.C. Mucosal immune responses to intestinal bacterial pathogens. Semin. Gastrointest. Dis. 7, 151-166 (1996).

12. Cox, D.S. \& Taubman, M.A. Oral induction of the secretory antibody response by soluble and particulate antigens. Int. Arch. Allergy Appl. Immunol. 75, 126 (1984).

13. Mackenzie, S.J. \& Halsey, J.F. Cholera toxin B subunit as a carrier protein to stimulate a mucosal immune response. J. Immunol. 133, 1818-1824 (1984).

14. Lycke, N. \& Holmgren, J. Strong adjuvant properties of cholera toxin on gut mucosal immune responses to orally presented antigens. Immunology 59, 301-308 (1986)

15. Jackson, R.J. et al. Optimizing oral vaccines: induction of systemic and mucosal
B-cell and antibody responses to tetanus toxoid by use of cholera toxin as an adjuvant. Infect. Immun. 61, 4272-4279 (1993).

16. Sun, J.B., Holmgren, J. \& Czerkinsky, C. Cholera toxin B subunit: An efficient transmucosal carrier-delivery system for induction of peripheral immunological tolerance. Proc. Natl. Acad. Sci. USA 91, 10795-10799 (1994).

17. Marinaro, M. et al. Use of intranasal IL-12 to target predominantly Th1 responses to nasal and Th2 responses to oral vaccines given with cholera toxin. J. Immunol. 162, 114-121 (1999).

18. Williams, N.A., Hirst, T.R. \& Nashar, T.O. Immune modulation by the cholera-like enterotoxins: from adjuvant to therapeutic. Immunol. Today 20, 95-101 (1999).

19. Xu-Amano, J. et al. Helper Th1 and Th2 cell responses following mucosal or systemic immunization with cholera toxin. Vaccine 12, 903-911 (1994).

20. Arakawa, T. et al. A plant-based cholera toxin B subunit-insulin fusion protein protects against the development of autoimmune diabetes. Nat. Biotechnol. 16, 934-938 (1998).

21. Pascual, D.W. et al. Expression of recombinant enterotoxigenic Escherichia coli colonization factor antigen I by Salmonella typhimurium elicits a biphasic T helper cell response. Infect. Immun. 67, 6249-6256 (1999).

22. Ball, J.M., Tian, P., Zeng, C.Q.Y., Morris, A.P. \& Estes, M.K. Age-dependent diarrhea induced by a rotavirus nonstructural glycoprotein. Science 272, 101-104 (1996).

23. Svennerholm, A.M. et al. Mucosal antitoxic and antibacterial immunity after cholera disease and after immunization with a combined B subunit-whole cell vaccine. J. Infect. Dis. 149, 884-893 (1984).

24. Horie, Y. et al. Diarrhea induction by rotavirus NSP4 in the homologous mouse model system. Virology 262 ,398-407 (1999).

25. Hajishengallis, G., Hollingshead, A.K., Koga, T. \& Russell, M.W. Mucosal immunization with a bacterial protein antigen genetically coupled to cholera toxin A2/B subunits. J. Immunol. 154, 4322-4332 (1995).

26. Dertzbaugh, M.T., Peterson, D.L. \& Macrina, F.L. Cholera toxin B-subunit gene fusion: structural and functional analysis of the chimeric protein. Infect. Immun. 58, 70-79 (1990)

27. Angel, J., Tang, B., Feng, N., Greenberg, H.B. \& Bass, D. Studies of the role for NSP4 in the pathogenesis of homologous murine rotavirus diarrhea. J. Infect. Dis. 177, 455-458 (1998).

28. Ishida, S.I., Feng, N., Gibert, J.M., Tang, B. \& Greenberg, H.B. Immune responses to individual rotavirus proteins following heterologous and homologous rotavirus infection in mice. J. Infect. Dis. 175, 1317-1323 (1997) 